\title{
Desarrollo de la fuerza muscular en niños como estrategia para disminuir el riesgo de enfermedad cardiometabólica
}

\author{
Patricio López-Jaramillo, 2, Daniel Dylan Cohen ${ }^{1,2}$ \\ Paul Anthony Camacho ${ }^{1}$, Jessica Hernández-Durán ${ }^{1,2}$, Diego Gómez-Arbeláez ${ }^{3}$
}

\author{
${ }^{1}$ Dirección de Investigaciones, Fundación Oftalmológica de \\ Santander (FOSCAL), Floridablanca, Santander, Colombia \\ ${ }^{2}$ Facultad de la Ciencias de la Salud, Universidad de Santander \\ (UDES), Bucaramanga, Santander, Colombia \\ ${ }^{3}$ Universidad Santiago de Compostela, España. \\ Autor para correspondencia \\ Patricio López-Jaramillo MD., PhD., FACP. \\ Director de Investigación, Desarrollo e Innovación Tecnológica \\ Fundación Oftalmológica de Santander-FOSCAL \\ Email: investigaciones@foscal.com.co \\ Director de Investigaciones \\ Facultad de Medicina - Universidad de Santander \\ Campus Universitario Lagos del Cacique, Bucaramanga-Santander, Colombia \\ Email: jplopezj@gmail.com
}

\section{Resumen}

$\mathrm{L}$ os factores de riesgo para las enfermedades cardiometabólicas (ECM) como la obesidad, la resistencia a la insulina y el síndrome metabólico tienen su inicio en la infancia. Existe evidencia de que la adiposidad infantil se relaciona con factores de riesgo para presentar enfermedad cardiovascular en la vida adulta, principalmente en la población hispana, en la cual se ha observado mayor vulnerabilidad para desarrollar enfermedades crónicas no transmisibles. Las altas tasas de morbimortalidad secundaria a enfermedades cardiovasculares en países de medianos y bajos ingresos económicos como Colombia, demanda profundizar en el estudio de los mecanismos que relacionan las bases biológicas y epigenéticas de la programación fetal y el riesgo de presentar ECM. Nosotros hemos publicado evidencias de que nuestra población tiene una alta sensibilidad para presentar inflamación de bajo grado y resistencia a la insulina a menores niveles de adiposidad visceral, asociada a una menor fuerza de empuñadura, la cual es un marcador del contenido de masa muscular. Proponemos que mejorar la condición física, sobre todo la capacidad aeróbica y la fuerza muscular, es una intervención efectiva para disminuir el riesgo de desarrollar enfermedad cardiovascular en niños y adolescentes colombianos, al disminuir la masa grasa, los marcadores de inflamación crónica de bajo grado y mejorar la cantidad y calidad de la masa muscular.

Palabras clave: programación fetal, obesidad infantil, inflamación crónica de bajo grado, actividad física, fuerza muscular.

\section{Abstract}

Risk factors for cardiometabolic diseases such as obesity, insulin resistance, and metabolic syndrome arise during childhood. There is evidence that adiposity in children is associated with risk factors for cardiovascular disease later in life, particularly among the Hispanic population, where vulnerability for the development of chronic non-communicable diseases is greater. The high mortality and morbidity rates of cardiovascular disease in middle-and low-income countries such as Colombia, makes it necessary to delve deeper into the mechanisms related to the biological and epigenetic basis of fetal programming, and the risk to develop cardiometabolic diseases. Based in our published studies, we have evidence that our population is highly prone to having chronic lowgrade inflammation and insulin resistance at lower levels of visceral adiposity, associated with a weaker handgrip, a muscle mass marker. Therefore, we propose that improving physical condition, more particularly aerobic capacity and muscle strength, is an effective intervention to decrease the risk of cardiovascular disease in Colombian children and adolescents, through a decrease in the fat mass, chronic lowgrade inflammation markers, and an improvement in both the quality and amount of muscle mass.

Key words: fetal programming, pediatric obesity, C-reactive protein, chronic low-grade inflammation, physical condition, muscle strength. 


\section{Introducción}

Los factores de riesgo para las enfermedades cardiometabólicas (ECM) tienen su inicio en la infancia ${ }^{(1)}$. La obesidad, la presión arterial elevada, la resistencia a la insulina, los triglicéridos elevados y el síndrome metabólico (SM) se presentan en niños y adolescentes y tienden a persistir hasta la vida adulta $^{(1)}$. Este fenómeno ha sido bien descrito en los países desarrollados pero es menos conocido en los países de bajos y medianos ingresos económicos ${ }^{(2)}$.

Dos estudios epidemiológicos internacionales en los cuales participó Colombia, el INTERHEART ${ }^{(3,4)}$ y el INTERSTROKE $^{(5)}$, demostraron que, en Latinoamérica, la obesidad abdominal es el factor con el mayor riesgo atribuible poblacional para la presencia de un primer infarto agudo de miocardio (IAM) y un primer accidente cerebrovascular (ACV) isquémico o hemorrágico. En verdad, la obesidad abdominal es un factor de riesgo independiente de gran importancia y un fácil predictor de desenlaces cardiovasculares ${ }^{(6)}$. La prevalencia de sobrepeso y obesidad ha aumentado exponencialmente en los últimos años, particularmente en población pediátrica, siendo en la actualidad un problema de salud pública reconocido internacionalmente. Se ha reportado que para el año 2010 hubo 42 millones de niños con sobrepeso en todo el mundo ${ }^{(7,8)}$. Esta problemática gana trascendencia si se tiene en cuenta que los niños con sobrepeso y obesidad tienden a seguir siendo obesos en la edad adulta y tienen más probabilidades de padecer, a edades más tempranas, enfermedades metabólicas y cardiovasculares $^{(7-9)}$. Uno de los posibles mecanismos que explican la relación entre obesidad y aumento del riesgo de enfermedad cardiovascular (ECV) tanto en niños como en adultos, es que el exceso de adiposidad es un determinante constante de inflamación crónica de bajo grado y de resistencia a la insulina, situaciones que tienen un papel fundamental en el desarrollo de aterosclerosis y $\operatorname{ECV}^{(10,11)}$.

En países de medianos y bajos ingresos económicos como Colombia, se ha visto que el incremento en la incidencia de SM, diabetes mellitus tipo 2 (DM2) y ECV se asocia a una posible predisposición genética de determinados grupos étnicos que al interactuar con factores medioambientales lleva a una mayor sensibilidad para desarrollar estas condiciones ${ }^{(8)}$. Un ejemplo de esto es la presencia de una mayor susceptibilidad en la población latinoamericana a desarrollar resistencia a la insulina e inflamación de bajo grado a menores perímetros abdominales o de índices de masa corporal ${ }^{(12)}$. Nosotros hemos propuesto que esta mayor susceptibilidad está relacionada con una respuesta adaptativa del feto a una deficiente nutrición materno-fetal ${ }^{(11,12)}$.

\section{Bases biológicas y epigenéticas}

La plasticidad en el desarrollo durante la programación fetal en mujeres gestantes malnutridas da lugar a una respuesta adaptativa del feto que permite el crecimiento de ciertos órganos clave como el sistema nervioso, en detrimento de otros como el tejido muscular, las nefronas, los cardiomiocitos y las células beta pancreáticas ${ }^{(11,12)}$. Si las condiciones de la vida intrauterina cambian durante la vida extrauterina, y se da un aumento exagerado de peso debido al consumo de alimentos densos en carbohidratos y grasas y a hábitos sedentarios, las adaptaciones implementadas durante el desarrollo fetal pueden resultar en un desequilibrio grasa/músculo, con individuos que presentan masa muscular disminuida y adiposidad aumentada, lo cual aumenta el riesgo de desarrollar SM, DM2 y ECV, independientemente de la relación peso/talla que alcance el individuo en la vida adulta (figura 1). El estudio INTERHEART, claramente demostró que individuos con un índice de masa muscular (IMC) menor de 20, pero que se encontraban en los quintiles más altos de adiposidad visceral (evaluada por la relación de los perímetros cintura/cadera) presentaron un aumentado riesgo para sufrir $\operatorname{IAM}^{(3,4)}$.

Las deficiencias nutricionales durante el periodo de plasticidad del desarrollo fetal pueden tener una influencia a largo plazo mediante la expresión o el silenciamiento de varios genes a través de mecanismos epigenéticos, cambios que son heredables y que resultan en aumento o disminución de la síntesis de proteínas tales como angiotensina II, leptina, y adiponectina, sustancias que se producen en el adipocito y que juegan un papel crítico en el desarrollo de resistencia a la insulina $\mathrm{y}$ de $\mathrm{ECM}^{(11-14)}$.

Actualmente ha quedado bien demostrado que existe una asociación entre el bajo peso al nacer y riesgo de desarrollar hipertensión arterial (HT), DM2 y ECV durante la vida adulta $^{(11,12,15-17)}$. Incluso esta relación podría ser independiente de otros factores de riesgo como tabaquismo, consumo excesivo de alcohol, obesidad y nivel socioeconómico. Aunque el bajo peso al nacer por sí solo no sería la causa de ECM, la asociación con una rápida recuperación del peso durante el periodo posnatal, el cual se acompaña de una distribución aumentada del contenido de grasa corporal y de bajos niveles de adiponectina, predisponen en el futuro a un mayor riesgo de obesidad y DM2 en la edad adulta y, en consecuencia, de un aumento en el riesgo de mortalidad por $\mathrm{ECV}^{(15-17)}$.

\section{Impacto de la industrialización, mecanización y urbanización}

Los rápidos procesos de industrialización, mecanización y urbanización que han experimentado los países latinoamericanos han ocasionado una acelerada transición hacia estilos de vida occidentales, cuyo impacto ha sido diferente en los diversos países de acuerdo con su desarrollo económico y al tiempo que ha transcurrido desde la exposición a estos cambios. En Colombia, gran parte de los habitantes de comunidades rurales han migrado hacia las ciudades como resultado del conflicto 
armado y el desplazamiento forzado, ocasionando importantes problemas socioeconómicos, cambios en los hábitos alimentarios en respuesta a la oferta cada vez mayor de alimentos procesados más económicos ${ }^{(18,19)}$. Además, el proceso de mecanización es notable en las actividades diarias de trabajo, y también en las de ocio, lo que ha conducido a estilos de vida sedentarios, principalmente en niños, quienes han remplazado los juegos tradicionales activos con alto requerimiento y consumo de energía, por juegos sedentarios frente a la televisión, los videojuegos y el internet. La población colombiana lleva poco tiempo expuesta a estos cambios, por lo que sus respuestas adaptativas a los excesos del estilo de vida moderno son todavía incipientes y esto podría explicar, al menos en parte, el aumento del riesgo de desarrollar $\operatorname{ECM}^{(18,19)}$ (figura 1).

Figura 1. Mecanismos biológicos y ambientales asociados con un mayor riesgo cardiovascular en la vida adulta

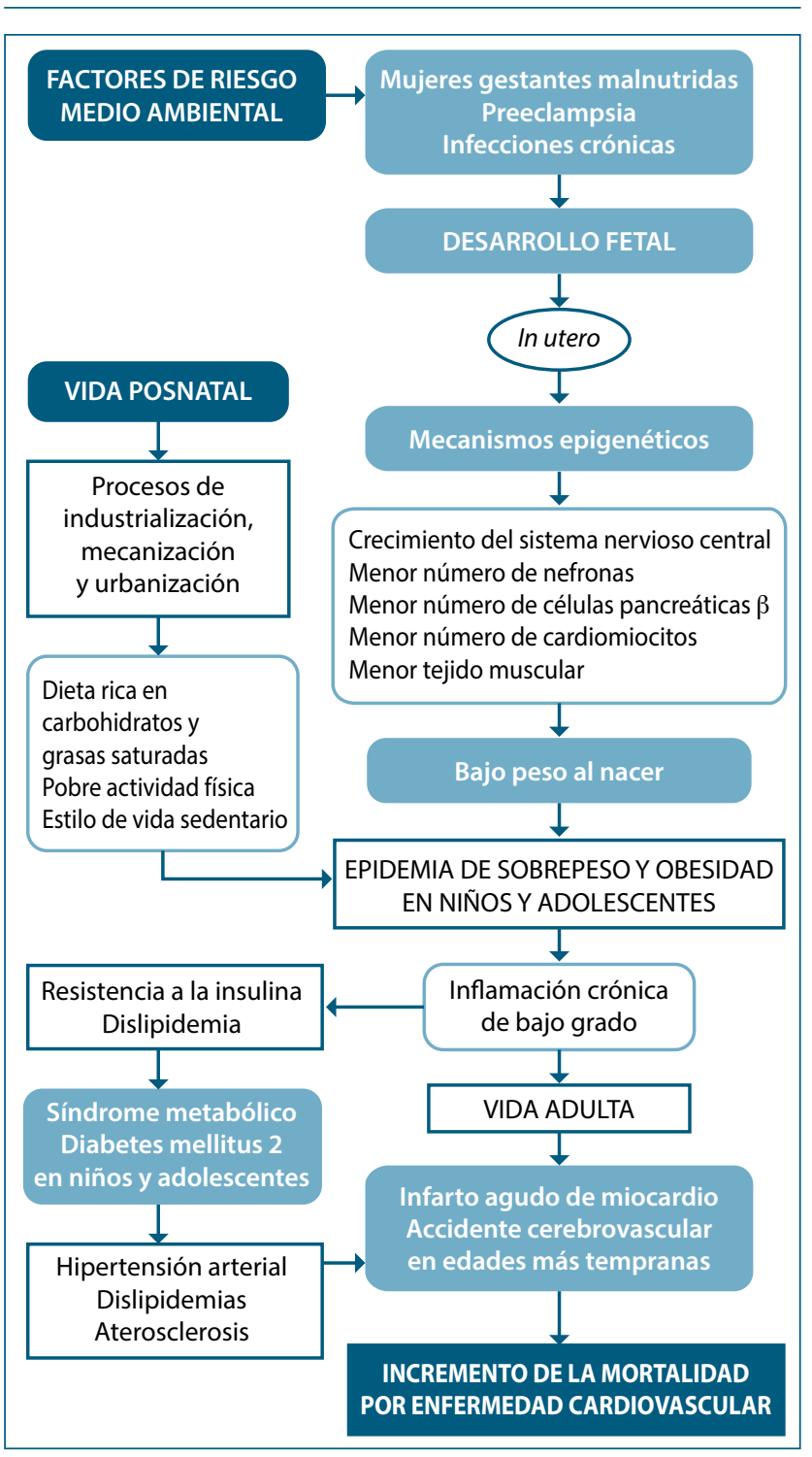

\section{El tejido adiposo como órgano endocrino}

El tejido adiposo no es sólo un depósito de energía, sino también un órgano secretor activo que libera a la circulación factores del complemento y citocinas proinflamatorias, particularmente interleucina 6 (IL-6) y factor de necrosis tumoral alfa (TNF- $\alpha$ ). Estas citocinas estimulan la producción hepática de proteína C-reactiva (PCR), un marcador inflamatorio de alta sensibilidad para inflamación sistémica y que se ha demostrado como un predictor de $\mathrm{ECV}^{(20)}$.

Estudios realizados en niños de los países desarrollados han demostrado que la asociación entre los factores de riesgo cardiovascular y las concentraciones de PCR es similar a la encontrada en adultos ${ }^{(21-23)}$. El estado de inflamación crónica de bajo grado se asocia con deterioro de la función del endotelio vascular, disminuida capacidad de producir óxido nítrico, y vasoconstricción periférica ${ }^{(24)}$.

Un estudio transversal realizado por nuestro grupo durante los años 2003-2004, que incluyó 325 niños y niñas escolares entre 9 y 11 años, de la ciudad de Bucaramanga ${ }^{(13)}$ demostró una correlación directa y progresiva entre niveles de adiposidad, concentraciones de PCR y presión arterial sistólica (PAS). Este estudio demostró que en nuestra población pediátrica existe una relación positiva entre niveles de adiposidad y marcadores de inflamación crónica de bajo grado, pero que el nivel de adiposidad a la cual se incrementa la PCR es menor en relación a lo reportado en niños de países desarrollados ${ }^{(20,21)}$. Estos resultados nos llevaron a proponer que nuestra población tiene una mayor sensibilidad para desarrollar inflamación de bajo grado a menores niveles de adiposidad visceral, situación que estaría relacionada con la contradicción entre la programación fetal y los rápidos cambios en los hábitos de vida que llevan a que los niños de países de medianos y bajos ingresos económicos, especialmente aquellos nacidos con bajo peso para la edad gestacional, presenten una composición corporal que favorece la acumulación de grasa a cambio de un menor desarrollo de masa muscular ${ }^{(8,19)}$.

En perspectiva de estudiar esta propuesta, desde el año 2011 iniciamos el desarrollo del proyecto ACFIES (asociación entre la capacidad cardiorrespiratoria, la fuerza muscular y la composición corporal con factores de riesgo metabólico en niños colombianos), con financiamiento de la Alcaldía de Bucaramanga y de la Fundación MAPFRE de España y con la colaboración de la Universidad Metropolitana de Londres y el Hospital Nacional de Niños de Washington D.C. Actualmente nos encontramos estudiando las variaciones epigenómicas del tejido graso en relación al IMC, a los niveles de adipocitocinas y a las interacciones con la medición de masa y función muscular en escolares de Bucaramanga, diferenciados por peso al nacer y nivel socioeconómico, en comparación con escolares de ascendencia hispana que viven en Washington y con niños caucásicos de la misma ciudad. En la tabla 1 se muestra la es- 
Tabla 1. Características demográficas y antropométricas de la población

\begin{tabular}{|c|c|c|c|c|}
\hline & $\begin{array}{c}\text { Total } \\
(n=669)\end{array}$ & $\begin{array}{c}\text { Niñas } \\
(\mathrm{n}=\mathbf{3 1 8})\end{array}$ & $\begin{array}{c}\text { Niños } \\
(\mathrm{n}=\mathbf{3 5 1})\end{array}$ & $\begin{array}{c}\text { Valor } \\
\text { P }\end{array}$ \\
\hline Edad (años) & $11,52 \pm 1,13$ & $11,52 \pm 1,10$ & $11,51 \pm 1,16$ & 0,838 \\
\hline Peso (kg) & $40,08 \pm 10,07$ & $40,33 \pm 9,77$ & $39,86 \pm 10,35$ & 0,636 \\
\hline Talla (m) & $1,45 \pm 0,09$ & $1,46 \pm 0,08$ & $1,44 \pm 0,09$ & 0,339 \\
\hline IMC $\left(\mathrm{kg} / \mathrm{m}^{2}\right)$ & $18,87 \pm 3,61$ & $18,81 \pm 3,52$ & $18,93 \pm 3,68$ & 0,004 \\
\hline $\begin{array}{l}\text { Circunferencia } \\
\text { abdominal }(\mathrm{cm})\end{array}$ & $65,95 \pm 9,73$ & $64,86 \pm 9,02$ & $66,92 \pm 10,24$ & 0,005 \\
\hline \multicolumn{5}{|l|}{ Estado Tanner } \\
\hline 1 & $368(55,1)$ & $149(47,0)$ & $219(62,4)$ & 0,004 \\
\hline 2 & $208(31,8)$ & $110(35,3)$ & $98(28,7)$ & 0,308 \\
\hline 3 & $78(11,7)$ & $53(16,7)$ & $25(7,1)$ & 0,249 \\
\hline \multicolumn{5}{|c|}{ Clasificación del peso (\%) } \\
\hline Bajo peso & $29(4,41)$ & $9(2,9)$ & $20(5,8)$ & 0,739 \\
\hline Peso normal & $479(69,9)$ & $240(77,2)$ & $239(68,9)$ & 0,041 \\
\hline Sobrepeso & $85(12,9)$ & $42(13,5)$ & $43(12,4)$ & 0,879 \\
\hline Obesidad & $65(8,8)$ & $20(6,4)$ & $45(12,9)$ & 0,436 \\
\hline
\end{tabular}

tadística descriptiva de los niños y niñas participantes en el estudio ${ }^{(25-26)}$.

Los primeros resultados obtenidos en este estudio demuestran que la prevalencia de sobrepeso y obesidad en el total de los participantes fue de $22,8 \%$ (12,92\% sobrepeso y $9,88 \%$ obesidad) (tabla 1). Observamos, además, una relación inversa entre la fuerza muscular ajustada por masa corporal con algunos factores de riesgo cardiovascular específicos como: triglicéridos, PCR, índice de resistencia a la insulina (índice HOMA), presión arterial sistólica (PAS) y presión arterial diastólica (PAD) y marcadores inflamatorios en los niños con sobrepeso y obesidad.

Adicionalmente, encontramos que a menores cuartiles de fuerza muscular se presentaron mayores valores en el puntaje de riesgo metabólico (figura 2), confirmando resultados previos que demuestran que en la población colombiana existe una mayor predisposición para presentar una respuesta inflamatoria con niveles de adiposidad más bajos que los reportados para la población caucásica, lo que posiblemente se asocia a largo plazo a un mayor riesgo de desarrollar ECV y enfermedad metabólica.

Estos resultados concuerdan con los reportados en otros estudios como el estudio AVENA (acrónimo en inglés para Diet and Assessment of the Nutritional Status in Adolescents) realizado con 2.859 adolescentes españoles en los que se encontró que bajos niveles de aptitud física están relacionados con mayor riesgo de enfermedades cardiometabólicas. Además, se ha observado que el mayor riesgo de desarrollar SM en individuos con bajo peso al nacer, desaparece en aquellos individuos que presentan un buen estado físico aeróbico o que realizan niveles significativos de actividad muscular vigorosa, lo que demuestra que, además de la cantidad de masa grasa y su distribución corporal, la cantidad y calidad de la masa muscular, así como el nivel de función muscular, podrían contribuir en la prevención de ECV en la vida adulta ${ }^{(27-29)}$.

Alrededor del mundo se han diseñado intervenciones de entrenamiento aeróbico y de fuerza muscular en niños y adolescentes, asociados a programas educativos sobre reducción en la ingestión de azúcar y aumento en el consumo de fibra vegetal. Estas intervenciones logran un impacto positivo en la sensibilidad a la insulina y los niveles de PCR séricos ${ }^{(30-32)}$, incluso sin cambios significativos en la composición corporal ${ }^{(30)}$.

Figura 2. Correlación entre la fuerza de empuñadura y el puntaje de riesgo metabólico ajustado por edad, sexo e IMC

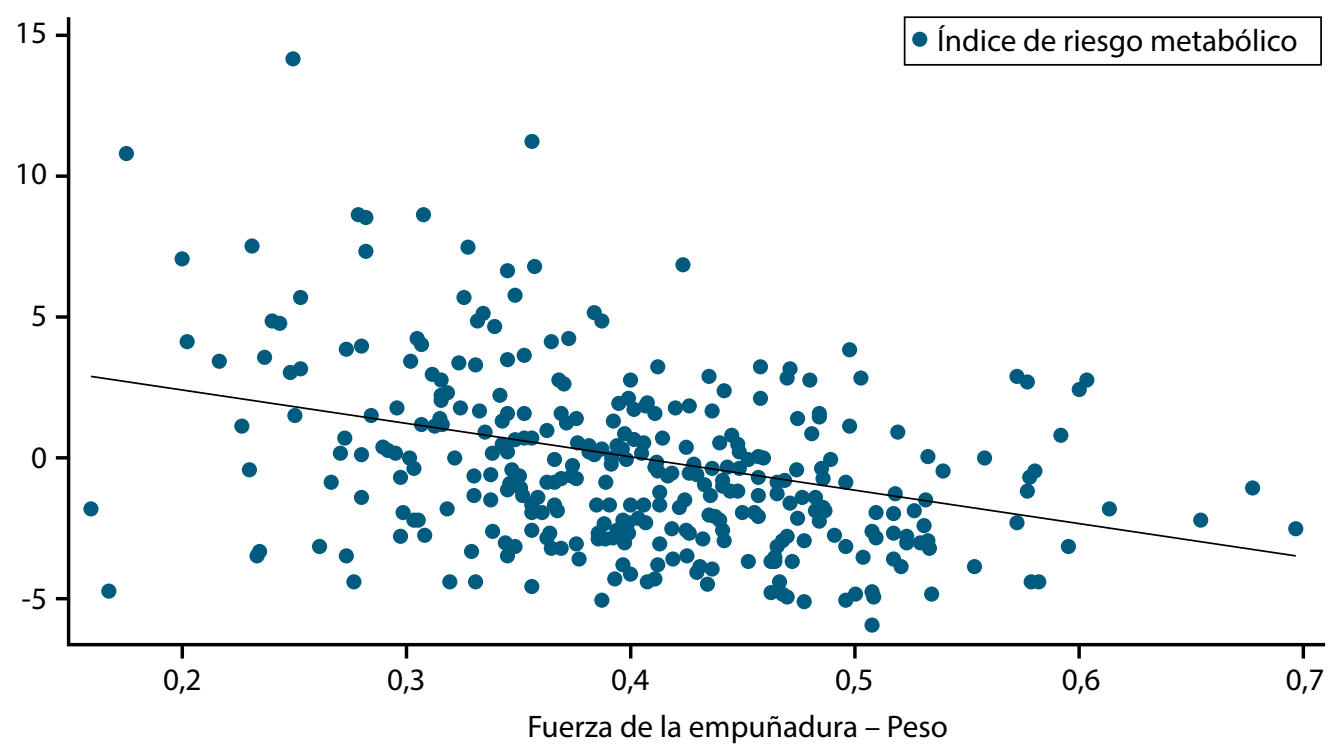


Un estudio realizado en adolescentes obesos latinoamericanos demostró que la implementación de una intervención de entrenamiento muscular de moderada intensidad, durante 16 semanas, logró mejorar la sensibilidad a la insulina y el control de la glucosa, por medio de una mayor captación de glucosa por el músculo esquelético y mecanismos enzimáticos a nivel hepático y muscular. Sin embargo, este estudio fue insuficiente para lograr cambios significativos en la reducción de la adiposidad y los marcadores inflamatorios ${ }^{(30)}$. Intervenciones a largo plazo (1 año de duración) en adultos, logran reducir significativamente los niveles de PCR plasmática asociados a un aumento en la masa muscular y reducción de la adiposidad, pero en la mayoría de los casos sin cambios en los niveles de IL-6 y TNF- $\alpha^{(32)}$. Por tanto, el entrenamiento de tipo aeróbico y de fuerza muscular mejora el perfil metabólico en individuos con resistencia a la insulina y DM2, incluso independientemente de los cambios en el peso corporal, especialmente en niños y adolescentes en donde se ha visto una mayor susceptibilidad ${ }^{(31-32)}$.

Mejorar la aptitud física a través de un programa de entrenamiento supervisado podría ser una intervención efectiva para disminuir los factores de riesgo de enfermedad cardiovascular en niños y adolescentes colombianos.

\section{Conclusiones y perspectivas}

Los datos presentados indican que las influencias ambientales, que se presentan desde el desarrollo fetal, ocasionan cambios epigenéticos que permanecen durante toda la vida e incluso son heredables. Un ambiente fetal adverso genera adaptaciones en el desarrollo y en la función de los tejidos humanos. Un ambiente fetal con deficientes aportes nutricionales de la madre, especialmente de proteínas, determina que el organismo en desarrollo favorezca el crecimiento del sistema nervioso a cambio de afectar el crecimiento de otros tejidos como el pancreático, renal y músculo-esquelético. Este recién nacido de bajo peso programado para resistir las deficiencias nutricionales se ve en la vida extrauterina expuesto a los efectos ocasionados por la rápida transición nutricional y estilos de vida sedentarios, que le produce una rápida ganancia de peso, la cual se basa principalmente en un aumento del tejido graso, que conduce a resistencia a la insulina e inflamación de bajo grado, dos elementos claves en el desarrollo de aterosclerosis y DM2. Actualmente hemos implementando un programa dirigido a mejorar la condición física de los niños incluidos en el estudio ACFIES, tanto de la capacidad aeróbica como de la fuerza muscular, para evaluar su impacto a corto plazo sobre marcadores de inflamación crónica de bajo grado, los niveles del índice HOMA y en los factores de riesgo cardio-metabólico. En un seguimiento a largo plazo, determinaremos si esta intervención disminuye el riesgo de desarrollar ECV en la vida adulta, especialmente en aquellos niños con bajo peso al nacer para la edad gestacional.

\section{Referencias}

1. Raitakari OT, Juonala M, Kähönen M, Taittonen L, Laitinen T, Mäki-Torkko N, et al. Cardiovascular risk factors in childhood and carotid artery intima-media thickness in adulthood: The cardiovascular risk in young Finns Study. JAMA. 2003; 290:2277-83.

2. Lopez-Jaramillo P, Camacho PA, Forero-Naranjo L. The role of environment and epigenetics in hypertension. Expert Rev Cardiovasc Ther 2013; 11 (11): 1455-7.

3. Yusuf S, Hawken S, Ounpuu S, Dans T, Avezum A, Lanas F, et al. Effect of potentially modifiable risk factors associated with myocardial infarction in 52 countries (the INTERHEART study): case-control study. Lancet. 2004; 364:93752.

4. Lanas F, Avezum A, Bautista LE, Diaz R, Luna M, Yusuf S, et al. Risk factors for acute myocardial infarction in Latin America: the INTERHEART Latin American study. Circulation. 2007; 115(9):1067-74

5. O’Donnell M, Xavier D, Liu L, Zhang H, Lopez-Jaramillo P, Yusuf S, et al. Risk factors for ischaemic and intracerebral haemorrhagic stroke in 22 countries (the INTERSTROKE study): a case-control study. Lancet. 2010; 376:112-23.

6. Rueda-Clausen CF, Lahera V, Calderon J, Bolívar IC, Castillo VR, López-Jaramillo P, et al. The presence of abdominal obesity is associated with changes in vascular function independently of other cardiovascular risk factors. Int J Cardiol. 2010; 139: 32-41

7. Rueda-Clausen C, Silva F, López-Jaramillo P. Epidemic of overweight and obesity in Latin America and the Caribbean. Int J Cardiol. 2008; 125:111-2.

8. Lopez-Jaramillo P, Lahera V, Lopez-Lopez J. Epidemic of cardiometabolic diseases: a Latin American point of view. Ther Adv Cardiovasc Dis. 2011; 5(2): 119-131.

9. Gómez-Arbeláez D, Camacho PA, Cohen DD, Alvarado-Jurado L, Duperly J, López-Jaramillo P, et al. Higher household income and the availability of electronic devices and transport at home are associated with higher waist circumference in Colombian children: The ACFIES study. Int J Environ Res Public Health. 2014; 11: 1834-1843.

10. García RG, Rincón MY, Arenas WD, Silva SY, Reyes LM, López-Jaramillo $\mathrm{P}$, et al. Hyperinsulinemia is a predictor of new cardiovascular events in Colombian patients with a first myocardial infarction. Int J Cardiol. 2011; 148: 85-90

11. López-Jaramillo P. Cardiometabolic Disease in Latin America: The Role of Fetal Programming in Response to Maternal Malnutrition. Rev Esp Cardiol. 2009;62:670-6.

12. López-Jaramillo P, López-López J. Fetal programming and cardiometabolic diseases: the role of angiotensin II and inflammation. Clin Invest Arterioscl. 2010;22(Supl 2):21-24.

13. López-Jaramillo P, Garcia G, Camacho P.A, Herrera E, Castillo V. Interrelationship between body mass index, C-re- 
active protein and blood pressure in a Hispanic pediatric population. Am J Hypertens. 2008; 21: 527-32.

14. López-Jaramillo P, Gómez-Arbeláez D, López-López J, LópezLópez C, Martínez-Ortega J, Gómez-Rodríguez A, et al. The role of leptin-adiponectin ratio in metabolic syndrome and diabetes. Horm Mol Biol Clin Invest. 2013; 18 (1): 37-45

15. Skinner M, Manikkam M, Guerrero-Bosagna C. Epigenetic transgenerational actions of endocrine disruptors. Reprod Toxicol. 2011; 31: 337-343.

16. Leach L, Mann G. Consequences of Fetal Programming for Cardiovascular Disease in Adulthood. Microcirculation. 2001; 18:253-5.

17. Skogen J, Øverland S. The fetal origins of adult disease: a narrative review of the epidemiological literature. J R Soc Med Sh Rep. 2012; 3:59.

18. López-Jaramillo P, Pradilla,L, Castillo V, Laherad V. Socioeconomic Pathology As a Cause of Regional Differences in the Prevalence of Metabolic Syndrome and Pregnancy-Induced Hypertension. Rev Esp Cardiol. 2007;60(2):168-78.

19. Lopez-Jaramillo P,Silva S, Rodríguez-Salamanca N, Duran A, Mosquera W, Castillo V. Are Nutrition-Induced Epigenetic Changes the Link Between Socioeconomic Pathology and Cardiovascular Diseases?. Am J Therap. 2008; 15: 362-72.

20. Rost NS, Wolf PA, Kase CS, Kelly-Hayes M, Silbershatz H, Massaro JM, et al. Plasma concentration of C-reactive protein and risk of ischemic stroke and transient ischemic attack: the Framingham study. Stroke. 2001; 32:2575-9.

21. Ford ES. C-Reactive protein concentration and cardiovascular disease risk factors in children: findings from the National Health and Nutrition Examination Survey 19992000. Circulation. 2003; 108:1053-8.

22. Gillum RF. Association of serum C-reactive protein and indices of body fat distribution and overweight in Mexican American children. J Natl Med Assoc 2003; 95:545-52.

23. Bautista LE, López-Jaramillo P, Vera LM, Casas JP, Otero AP, Guaracao AI. Is C-reactive protein an independent risk factor for essential hypertension? J Hypertens. 2001; 19:857861.
24. García RG, Zarruk JG, Barrera C, Pinzón A, Trillos E, LópezJaramillo P, et al. Plasma nitrate levels and flow-mediated vasodilation in untreated major depression. Psychosom Med. 2011; 73: 344-349. .

25. Cohen DD, Gómez-Arbeláez D, Camacho PA, Pinzón S, Hormiga C, et al. Low Muscle Strength Is Associated with Metabolic Risk Factors in Colombian Children: The ACFIES Study. PLoS ONE. 2014; 9(4): e93150.

26. López-Jaramillo P, Gómez-Arbeláez D, Cohen DD, Camacho PA, Rincón-Romero K, Hormiga C, et al. Asociación entre obesidad y baja capacidad muscular y función cardiorrespiratoria, factores de riesgo cardiometabólico en niños colombianos. Trauma. 2013; 24: 17-23.

27. Steene-Johannessen J, Anderssen SA, Kolle E, Andersen LB. Low muscle fitness is associated with metabolic risk in youth. Med Sci Sports Exerc. 2009; 41: 1361-7.

28. Martínez-Gómez D, Gómez-Martínez S, Ruiz JR, Diaz LE, Ortega FB, et al. Objectively-measured and self-reported physical activity and fitness in relation to inflammatory markers in European adolescents: the HELENA Study. Atherosclerosis. 2012; 221: 260-7.

29. Benson AC, Torode ME, Singh MA. Muscular strength and cardiorespiratory fitness is associated with higher insulin sensitivity in children and adolescents. Int J Pediatr Obes. 2006; 1: 222-31.

30. Hasson R, Adam T, Davis J, Kelly L, Ventura E, Byrd-Williams C, et al. Randomized Controlled Trial to Improve Adiposity, Inflammation, and Insulin Resistance in Obese AfricanAmerican and Latino Youth. Obesity (Silver Spring). 2012; 20(4): 811-818.

31. Dâmaso AR, Campos RM, Caranti DA, de Piano A, Fisberg $\mathrm{M}$, Foschini D, et al. Aerobic plus resistance training was more effective in improving the visceral adiposity, metabolic profile and inflammatory markers than aerobic training in obese adolescents. J Sports Sci. 2014; 14:1-11.

32. Calle C, Fernandez M. Effects of resistance training on the inflammatory response. Nutr Res Pract. 2010;4(4):259269. 